\title{
Opioid-free anaesthesia in three dogs
}

\author{
Donna M. White*, Alastair R. Mair and Fernando Martinez-Taboada \\ Department of Anaesthesia and Analgesia, Veterinary Teaching Hospital, University of Sydney, Evelyn Williams \\ Building B10, 65 Parramatta Road, Camperdown, NSW. 2050. Australia
}

\begin{abstract}
Opioid-free anaesthesia (OFA) is a relatively new and growing field in human medicine. There are multiple motivations behind this emerging practice with the recognition of several serious potential opioid-related adverse effects including opioid induced hyperalgesia, opioid tolerance and immunomodulatory effects of opioids. Opioids have long been the mainstay of veterinary anaesthesia and pain management practice. The feasibility of OFA in veterinary patients is presented here. A case series of three dogs that underwent OFA for canine ovariohysterectomy is reported. The authors conclude OFA is possible in veterinary medicine; however the move away from the familiar effects of opioids perioperatively is challenging. Gaining experience with these types of protocols for standard procedures in healthy animals, such as neutering, will provide the anaesthetist with the building blocks for more invasive surgeries.
\end{abstract}

Keywords: Anaesthesia, Analgesia, Dog, Pain.

\section{Introduction}

Opioids have long been a cornerstone of pain management in both human and veterinary anaesthesia. They provide analgesia, sedation and anaesthetic sparing effects, and often contribute significantly to haemodynamic stability during surgery and anaesthesia. Opioid use is not without side effects that can include well recognised clinically significant respiratory depression, ileus and potential for development of addiction and tolerance in both humans (Ronald and Kissin, 1998) and animals (Martin and Eades, 1961; Kissin et al., 1991). Currently there is a growing body of evidence, from both human and animal studies, supporting a condition of opioidinduced hyperalgesia OIH (Lee et al., 2011; Pasero and McCaffery, 2012). There has been a recognition of the negative immunomodulatory effects of some opioids (Sacerdote, 2006; Odunayo et al., 2010; Levite, 2012) and even an association of increased rates of cancer recurrence with opioid use (Cata et al., 2016).

Opioid-induced hyperalgesia can occur in a subset of patients, for currently undefinable reasoning. Increased post-operative pain scores and opioid requirements have been reported in people following administration of a single opioid-containing anaesthetic (Sukhani et al., 1996; Chia et al., 1999; Guignard et al., 2000; Bakan et al., 2015). Administration of opioids initially produces analgesia but after a period of time hyperalgesia may be induced (Angst and Clark, 2006). While the condition itself is still poorly understood, the seriousness of a hyperalgesic state for postoperative patients has provided the motivation for the fast growing practice of opioid-free anaesthesia (OFA) in human medicine. Additional reported benefits of OFA include reduced post-operative opioid requirements (Feld et al., 2003; Mansour et al., 2013; Bakan et al., 2015), reduced incidence of post-operative nausea and vomiting (Mather and Peutrell, 1995; Callesen et al., 1999; Ziemann-Gimmel et al., 2014; Bakan et al., 2015), reduced intraoperative hypotension (Jagannathan et al., 2007; Bakan et al., 2015), and reduced post-operative sedation levels (Feld et al., 2003). Opioids provide the mainstay of analgesia in a significant proportion of veterinary anaesthesia. Veterinarians are familiar with their use and perioperative effects. The use of OFA provides a difficult challenge with loss of this familiarity. However it is likely there is a subset of veterinary patients that are also susceptible to OIH and arguable that our oncologic and immunodeficient patients may also benefit from OFA (Celerier et al., 2000; Cabanero et al., 2009). OFA highlights the need for a multimodal drug approach to ensure the patient receives an opioidfree, but not analgesic-free, anaesthetic and postanaesthetic experience. This report presents three cases that underwent successful OFA, with a protocol the authors consider an acceptable baseline for more invasive surgeries, and with a potential for prospective randomized studies.

\section{Case details}

All cases discussed were presented to the University Veterinary Teaching Hospital Sydney (UVTHS), for routine ovariohysterectomy, through the teaching neuter program. 


\section{Case 1}

A 6-month-old Bull Terrier was presented for ovariohysterectomy. The owner reported no current health concerns but noted a previous episode of conjunctivitis one month prior to presentation. The dog's physical examination findings were unremarkable. Her demeanour was noted to be quiet and friendly.

\section{Case 2}

A 2-year-old Shetland Sheepdog presented for ovariohysterectomy. The dog was current on her vaccination schedule with no ill-health reported by the owner. Physical exam of the dog revealed a grade II/VI heart murmur, with point of maximum intensity over the left heart base. The owner declined cardiac work up, however, reported no clinical signs of cardiac disease. The dog's demeanour was noted to be bright, alert and responsive.

\section{Case 3}

A 5-year-old Labrador was presented for ovariohysterectomy. The owner reported no health concerns. The dog had whelped three months prior to presentation. Physical examination was unremarkable. The dog was noted to be very timid and nervous of people.

\section{Procedures}

Dogs underwent the standard pre-anaesthetic protocol of the teaching hospital neuter clinic, performed by final year veterinary students during their anaesthesia clinical training rotation. This included physical examination, basic pre-anaesthetic blood testing (PCV/TP), preoperative behaviour scoring (Romano et al., 2015) and assignment of ASA health status score (Table 1).

Perioperative patient assessments included sedation scoring (Gurney et al., 2009), recovery scoring (Becker et al., 2013) and post-operative pain scoring (Reid et al., 2007), performed by students with guidance from an anaesthetist (Table 1).

The UVTHS neuter clinic protocol dictates that sedation scoring is performed immediately prior to induction. Recovery scoring is performed immediately after extubation. Post-operative pain scoring is initially performed when the dog is extubated and responsive, and thereafter hourly for three hours (by anaesthesia students). Once transferred to recovery ward dogs' pain was scored every four hours (by recovery ward nursing staff) until discharge time (the following morning).

The same anaesthetist managed all three cases. OFA was achieved with a pre-anaesthetic medication of varying doses of medetomidine (Domitor, Pfizer Animal Health, Australia), ketamine (Ceva Ketamine, Ceva Animal Health Pty Ltd, Australia) and acepromazine (A.C.P.2, Delvet, Australia). All drugs were mixed in a single syringe and administered intramuscularly.
Table 1. Pre-anaesthetic blood testing (PCV/TP), ASA health status score, perioperative sedation scoring, intraoperative anaesthetic complications, post-operative recovery scoring, and post- operative pain scoring for each case.

\begin{tabular}{lccc}
\hline Parameter & Case 1 & Case 2 & Case 3 \\
\hline PCV/TP & $51 / 66$ & $43 / 60$ & $58 / 62$ \\
ASA & 1 & 2 & 1 \\
Sedation Score (/15) & 10 & 6 & 6 \\
Anaesthetic & H. & SR (surgical). SR (surgical). \\
complications & H. & H. \\
Recovery Score (/4) & 1 & 1 & 1 \\
PS 1 (/25) (alert and & 1 & 3 & 1 \\
response) & 1 & 8 & $2(4 \mathrm{pm})$ \\
PS 2 (+60mins) & 3 & 4 & 2 \\
PS 3 (+120 mins) & 2 & $0(4 \mathrm{pm})$ & 2 \\
PS 4 (+180mins) & 1 & 0 & 2 \\
PS 4pm & 2 & 0 & 6 \\
PS 8pm & 1 & 1 & 2 \\
PS12 midnight & 2 & 0 & 2 \\
PS 4am & 0 & 0 & 2 \\
PS 8am & sympathetic & response; & (H): \\
\hline (SR): intraoperativermia \\
(<37 ${ }^{\circ}$ C); (PS): Pain score.
\end{tabular}

Anaesthesia was induced intravenously with alfaxalone (Alfaxan, Jurox, Australia) or alfaxalone and lidocaine (Lignocaine Injection 2\%, Pfizer Animal Health, Australia) co-induction. Anaesthesia was maintained with Isoflurane (Isothesia, Henry Schein, Australia) in oxygen.

Medetomidine and ketamine infusions were administered intraoperatively for analgesia and minimum alveolar concentration (MAC) sparing effects to improve the dogs' haemodynamics. Sympathetic response (dramatic or sustained increases in heart rate and blood pressure) was viewed as light plane of anaesthesia for the level of surgical stimulation and was treated with either adjustment in isoflurane concentration or intraoperative injection of analgesia (ketamine or lidocaine). Transversus abdominis plane (TAP) regional anaesthesia with bupivacaine (Bupivacaine Injection BP 0.5\%, Pfizer Animal Health, Australia) $4 \mathrm{mg} / \mathrm{kg}$ was performed in all cases. Carprofen (Carprieve, Norbrook Laboratories, Australia Ltd, Australia) $4 \mathrm{mg} / \mathrm{kg}$ was administered subcutaneously and paracetamol (Paracetamol Kabi, Fresenius Kabi Australia Pty Ltd, Australia) $10 \mathrm{mg} / \mathrm{kg}$ intravenously during the preoperative or intraoperative period.

Atracurium (Atracurium Besylate Injection, Hospira Australia Pty Ltd, Australia) was administered to one of the three cases (Case 2) as required to assist mechanical ventilation. All three cases were monitored 
with use of pulse oximetry, capnography, non-invasive (appropriately sized cuff placed on antebrachium) or invasive blood pressure (arterial catheter placed in dorsal pedal artery), volatile anaesthetic monitoring, intraoperative oesophageal and post-operative rectal temperatures. Animals were visually monitored continuously in recovery until alert and responsive, standing and having a rectal temperature above 37 degrees Celsius. Active warming was provided with a warm air blanket (Warm Air Blanket, Darvall Vet, Australia).

The protocol was varied with each case as indicated by individual patient factors and for improvement of the overall technique (Table 2).

Table 2. OFA protocol for each case.

\begin{tabular}{|c|c|c|c|}
\hline Drugs & Case 1 & Case 2 & Case 3 \\
\hline $\begin{array}{l}\text { Medetomidine (ug/kg) } \\
\text { IM }\end{array}$ & 10 & 5 & 10 \\
\hline Ketamine $(\mathrm{mg} / \mathrm{kg}) \mathrm{IM}$ & 2 & 2 & 2 \\
\hline $\begin{array}{l}\text { Acepromazine }(\mathrm{mg} / \mathrm{kg}) \\
\text { IM }\end{array}$ & 0.02 & 0.02 & 0.02 \\
\hline Alfaxalone $(\mathrm{mg} / \mathrm{kg}) \mathrm{IV}$ & 0.4 & 2 & $2.7 \mathrm{mg} / \mathrm{kg}$ \\
\hline Lidocaine $(\mathrm{mg} / \mathrm{kg}) \mathrm{IV}$ & 1 & - & - \\
\hline Isoflurane (ET\%) & $0.8-1.4$ & $0.9-1.1$ & $0.9-1.3$ \\
\hline $\begin{array}{l}\text { Ketamine CRI } \\
\text { (ug/kg/min) } \\
\text { /total dose }(\mathrm{mg} / \mathrm{kg})\end{array}$ & $10 / 0.8$ & $5-10 / 1.2$ & $10 / 1.3$ \\
\hline $\begin{array}{l}\text { Lidocaine CRI } \\
\text { (ug/kg/min) } \\
\text { /total dose }(\mathrm{mg} / \mathrm{kg})\end{array}$ & $50 / 1.3$ & - & - \\
\hline $\begin{array}{l}\text { Dex/Medetomidine CRI } \\
\text { (ug/kg/hr) /total dose } \\
(\mathrm{ug} / \mathrm{kg})\end{array}$ & $\mathrm{M} 2 / 2.4$ & M2/4.6 & $\mathrm{M} 2 / 4.4$ \\
\hline $\begin{array}{l}\text { Ketamine bolus IV } \\
\text { /(total dose } \mathrm{mg} / \mathrm{kg} \text { ) }\end{array}$ & $\mathrm{N}$ & $\mathrm{N}$ & $\mathrm{Y}(0.5)$ \\
\hline $\begin{array}{l}\text { Lidocaine bolus IV } \\
\text { /(total dose } \mathrm{mg} / \mathrm{kg} \text { ) }\end{array}$ & $\mathrm{N}$ & $\mathrm{N}$ & $\mathrm{Y}(2)$ \\
\hline TAP block & $\mathrm{Y}$ & $\mathrm{Y}$ & $\mathrm{Y}$ \\
\hline
\end{tabular}

Case 1 received the highest sedation score of 10/15. It should be noted Case 1 was assessed to be quiet and friendly preoperatively compared with the following two cases that were noted to be bright, alert and responsive or nervous and timid preoperatively, and as such this difference in patient factors may have contributed to the heavier sedation level achieved. Case 1 utilised lidocaine as a co-induction agent and this likely contributed to a dose sparing effect of alfaxalone. For Case 2, the premedication dose of medetomidine was half that of Case 1 with no change to the dosing of ketamine or acepromazine. Although heavy sedation was achieved (sedation score 9/15) the dog was also noted to be dysphoric. Subsequent to this a high dose of alfaxalone was required to induce anaesthesia. Lower end-tidal isoflurane concentrations were used to maintain anaesthesia with no intraoperative analgesia boluses required.

However, Case 2 had the only pain score $>5 / 24$ in the first three hours post-operatively. Case 2 was administered methadone analgesia however the dog's pain score did not improve with this. The increased score was deemed to be primarily anxiety based and was successfully treated with acepromazine $(0.01 \mathrm{mg} / \mathrm{kg})$ intravenously. In an attempt to eliminate the possibility of dysphoria Case 3 received a higher dose of medetomidine, as also previously given to Case 1. Although the dog did not appear dysphoric she did show muscle rigidity, suspected secondary to ketamine administration. Subsequently a higher dose of alfaxalone was required to induce anaesthesia. Postoperative pain scoring for Case 3 remained low with no post-operative opioids required.

Anaesthetic complications included increases in heart rate and blood pressure, and hypothermia $\left(<37^{\circ} \mathrm{C}\right)$. No episodes of intraoperative hypotension occurred. Hypothermia resolved within two hours postoperatively in all dogs. The recovery scores of all dogs reflected excellent post-operative recoveries.

\section{Discussion}

OFA is possible for dogs undergoing routine ovariohysterectomy. OFA was achieved in a series of three cases using a multimodal drug approach including medetomidine, ketamine, carprofen, paracetamol and bilateral TAP regional anaesthesia with bupivacaine. The long-standing use of opioids in veterinary anaesthesia has led to a familiarity of their effects during anaesthesia. Opioids are potent analgesics with significant MAC sparing effects. The UVTHS neuter clinic utilises the MAC-sparing effects of opioids in the vast majority of anaesthetics. Loss of this familiarity with increasing use of OFA has brought with it several challenges. The use of a multidrug approach was deemed essential for OFA to ensure adequate analgesia. The protocol presented was developed based on currently used opioid-free protocols in human anaesthesia (Bakan et al., 2015; Mulier, 2016). With each case came increased familiarity of the drug combinations resulting in increased competence in performing OFA.

Medetomidine is a selective alpha-2 agonist drug providing analgesia, sedation and sympatholysis with significant opioid sparing effects (Blaudszun et al., 2012). Alpha-2 agonist analgesic doses can be variable and the duration of analgesia is unreliable (Sinclair, 2003). Medetomidine can be used in combination with an opioid, rather than a potential substitute, to improve its analgesic effects (Kuo and Keegan, 2003), providing a potential drawback of OFA. Alpha-2 agonist drugs are considered core components in many human OFA protocols (Bakan et al., 2015; Mulier, 2016). Ketamine is a dissociative agent with NMDA receptor 
antagonism and sympathomimetic effects (Peltoniemi et al., 2016). At low doses it provides analgesia, while also providing anaesthesia at high doses (Peltoniemi et al., 2016). It is thought to provide less intense analgesia than pure- $\mu$ opioids and is often used in combination with an opioid for multimodal analgesic effects (Carstensen and Moller, 2010; Gutierrez-Blanco et al., 2015).

Ketamine is a common component of OFA in people and has even been shown to reduce the development of $\mathrm{OIH}$ if administered prior to opioid treatment in animals (Celerier et al., 2000) and humans (Hong et al., 2011; Kaur et al., 2015). The dysphoria reported for Case 2 after premedication was believed to be secondary to ketamine administration. Continuation of ketamine intraoperatively may also have contributed to the post-operative anxiety shown by the dog. Subanaesthestic doses of ketamine have been shown to cause adverse behaviour changes in humans (Krystal et al., 1994).

Case 3 experienced muscle rigidity following premedication and this was again considered a side effect of ketamine administration. These side effects are potential drawbacks of utilizing ketamine within opioid free protocols. Ketamine is often omitted or used at lower doses when combined with opioids.

Intravenous lidocaine has been shown to have analgesic and anti-inflammatory effects (Rang et al., 2012). Intraoperative intravenous lidocaine infusion can reduce inhalational anaesthetic requirements, reduce post-operative pain scores and opioid requirements (McCarthy et al., 2010).

Carprofen and paracetamol have been used as adjunct analgesics in these cases. Although they do not contribute an anaesthetic sparing effect they add to the multimodal approach that is essential when considering OFA. Carprofen is a non-steroidal anti-inflammatory analgesic with COX-2 inhibitory effects. Carprofen has been shown to be safe and efficacious when given preoperatively to healthy animals undergoing fracture repair (Bergmann et al., 2005).

Paracetamol is a centrally acting analgesic with mild to moderate effects (Rang et al., 2012). Paracetamol is not commonly used in veterinary medicine compared to its use in human medicine, however it is widely used at the UVTHS. Species variation in use, and specifically acute toxicity effects in felines, and a lack of familiarity are likely significant contributors to the reduced use of paracetamol in veterinary medicine. Although not often used on its own paracetamol has been shown repeatedly to provide significant opioid-sparing effects in humans (Korpela et al., 1999; Remy et al., 2005; Sinatra et al., 2005; Maund et al., 2011). Mburu et al. (1988) reported a $33 \%$ reduction in post-operative surgical site swelling and $47 \%$ reduction in pain in dogs treated with paracetamol compared with placebo after undergoing experimental forelimb surgery. Paracetamol was well tolerated in the cases presented with no adverse effects seen.

When preparing an OFA technique, a great amount of effort must go into ensuring an effective multimodal approach is achieved, as one of the potential drawbacks associated with the practice of OFA is inadequate analgesia. Traditional opioid anaesthetic (OA) techniques for routine ovariohysterectomies at UVTHS involve the use of opioids and non-steroidal antiinflammatories. Premedication is usually achieved with medetomidine and/or acepromazine, as done in this OFA technique. The addition of intraoperative constant rate infusions of medetomidine and ketamine, and lidocaine boluses were the mainstay of the OFA techniques presented. Regional anaesthesia in the form of TAP block with bupivacaine was viewed as only adjunctive analgesia and believed to likely be of greatest benefit post-operatively for the laparotomy wound.

There were notable differences of OFA to OA. The first occurred with premedication. A standard premedication for OA at the UVTHS involves use of a sedative and opioid. For these OFA cases the opioid was essentially substituted with ketamine and medetomidine. The dose of medetomidine required to achieve the same level of sedation that would be expected when combined with morphine was approximately double than when combined with ketamine and midazolam.

This highlighted the clinically significant synergistic effects of adding an opioid to medetomidine, especially when considering the lower level of sedation that is expected when morphine is given on its own. Acepromazine was administered to Case 2 postoperatively after the dog showed signs of anxiety on recovery and following no response to methadone analgesia trial bolus. It is unlikely the sedative effects of the ketamine and medetomidine were remaining during recovery, with acepromazine the only longacting drug utilized in this protocol. This varies significantly from traditional OA techniques where longer acting opioids, such as morphine and methadone, are often given as part of the premedication, providing ongoing sedative effects and contributing to recovery quality.

The second notable difference, and arguably the most clinically relevant, was a lack of hypotension during OFA. Hypotension is one of the most common complications of anaesthesia (Gaynor et al. 1999). Increase in blood pressure can be a sign of sympathetic response to surgical stimulation. Initial management of dramatic or sustained increases in blood pressure included increasing anaesthetic depth or provision of an analgesic dose of ketamine intravenously. However a lack of response to such measures and lack of physical 
indicators of light plane of anaesthesia (eye position, jaw tone) resulted in recognition of blood pressure values in the high normal range to be secondary to our combination of anaesthetic drugs. Increasing the depth of anaesthesia of the dogs at this time resulted in physical indicators of deep anaesthesia (centrally placed eyes and lack of jaw tone).

The most likely contributor was the peripheral vascular effects of medetomidine with subsequent vasoconstriction. These results are consistent with those of Bakan et al. (2015) who compared OFA with propofol, dexmedetomidine and lidocaine infusions with opioid anaesthesia of propofol and remifentanil infusions and found the OFA group had only 3\% of patients requiring use of ephedrine for treatment of hypotension compared with $20 \%$ of the OA group. Furthermore $28 \%$ of the OFA group actually required treatment for hypertension while none of the OA group did. As the authors' experience with OFA increased the recognition of increased blood pressure values as drug effects lead to greater comfort level with management of the anaesthesia and subsequently comfort in appropriately using lower inhalational levels.

All dogs had a smooth initial recovery with low recovery scores at extubation. Analgesia was administered to Case 2 to ensure pain was not a component of the anxious behaviour shown postoperatively (with no response). The lack of pain in all dogs on recovery was somewhat unexpected with an almost expected pain response in the face of opioid omission.

Use of OFA will continue to be a growing field in human medicine and will likely be of benefit to a particular subset of veterinary patients. Development of OFA protocols for more invasive, advanced surgeries can occur once the anaesthetist is comfortable performing OFA in less invasive, commonly performed surgeries such as ovariohysterectomy.

\section{Conflict of interest}

The Authors declare that there is no conflict of interest.

\section{References}

Angst, M.S. and Clark, J.D. 2006. Opioid-induced hyperalgesia; A qualitative systemic review. Anesthesiology 104, 570-587.

Bakan, M., Umutoglu, T., Topuz, U., Uysal, H., Bayram, M., Kadioglu, H. and Salihoglu, Z. 2015. Opioid- free total intravenous anaesthesia with propofol, dexmedetomidine and lidocaine infusions for laparoscopic cholecystectomy: a prospective, randomised, double-blind study. Rev. Bras. Anestesiol. 65(3), 191-199.

Becker, W.M., Mama, K.R., Rao, S., Palmer, R.H. and Egger, E.L. 2013. Prevalence of dysphoria after fentanyl in dogs undergoing stifle surgery. Vet. Surg. 42, 302-307.
Bergmann, H.M.L., Nolte, I.J.A. and Kramer, S. 2005. Effects of preoperative administration of carprofen on renal function and hemostasis in dogs undergoing surgery for fracture repair. Am. J. Vet. Res. 66(8), 1356-1363.

Blaudszun, G., Lysakowski, C., Elia, N. and Tramer, M.R. 2012. Effect of perioperative systemic $\alpha 2$ agonists on postoperative morphine consumption and pain intensity: systematic review and metaanalysis of randomized controlled trials. Anesthesiology 116(6), 1312-1322.

Cabanero, D., Campillo, A., Celerier, E., Romero, A. and Puig, M.M. 2009. Pronociceptive effects of remifentanil in a mouse model of postsurgical pain: effect of a second surgery. Anesthesiology 111, 1334-1345.

Callesen, T., Schouenborg, L., Nielsen, D., Guldger, H. and Kehlet, H. 1999. Combined epidural-spinal opioid-free anaesthesia and analgesia for hysterectomy. Br. J. Anaesth. 82(6), 881-885.

Carstensen, M. and Moller, A.M. 2010. Adding ketamine to morphine for intravenous patientcontrolled analgesia for acute postoperative pain: a qualitative review of randomized trials. $\mathrm{Br}$. J. Anaesth. 104(9), 401-406.

Cata, J.P., Bugada, D., Marchesini, M., De Gregor, M. and Allegri, M. 2016. Opioids and cancer recurrence: a brief review of the literature. Can. Cell Microenviron. 2016; 3: e1159. doi: 10.14800/ccm.1159.

Celerier, E., Rivat, C., Jun, Y., Laulin, J.P., Larcher, A., Reynier, P. and Simonnet, G. 2000. Long-lasting hyperalgesia induced by fentanyl in rats: Preventative effect of ketamine. Anesthesiology 92, 465-471.

Chia, Y.Y., Liu, K., Wang, J.J., Kuo, M.C. and Ho, S.T. 1999. Intraoperative high dose fentanyl induces postoperative fentanyl tolerance. Can. J. Anaesth. 46, 872-877.

Feld, J.M., Laurito, C.E., Beckerman, M., Vincent, J. and Hoffman, W.E. 2003. Non-opioid analgesia improves pain relief and sedation after gastric bypass surgery. Can. J. Anaesth. 50(4), 336-341.

Gaynor, J.S., Dunlop, C.I., Wagner, A.E., Wertz, E.M., Golden, A.E. and Demme, W.C. 1999. Complications and mortality associated with anesthesia in dogs and cats. J. Am. Anim. Hosp. Assoc. 35(1), 13-17.

Guignard, B., Bossard, A.E., Coste, C., Sessler, D.I., Lebrault, C., Alfonsi, P., Fletcher, D. and Chauvin, M. 2000. Acute opioid tolerance: Intraoperative remifentanil increases postoperative pain and morphine requirement. Anaesthesiology 93, 409417.

Gurney, M., Cripps, P. and Mosing, M. 2009. Subcutaneous pre-anaesthetic medication with 
acepromazine-buprenorphine is effective as and less painful than the intramuscular route. J. Small Anim. Pract. 50(9), 474-477.

Gutierrez-Blanco, E., Victoria-Mora, J.M., Ibancovichi-Camarillo, J.A., Sauri-Arceo, C.H., Bolio González, M.E., Acevedo-Arcique, C.M., Marin-Cano, G. and Steagall, P.V. 2015. Postoperative analgesic effects of either a constant rate infusion of fentanyl, lidocaine, ketamine, dexmedetomidine, or the combination lidocaineketamine-dexmedetomidine after ovariohysterectomy in dogs. Vet. Anaesth. Analg. 42(3), 309-318.

Hong, B.H., Lee, W.Y., Kim, Y.H., Yoon, S.H. and Lee, W.H. 2011. Effects of intraoperative low dose ketamine on remifentanil-induced hyperalgesia in gynecologic surgery with sevoflurane anesthesia. Korean J. Anesthesiol. 61(3), 238-243.

Jagannathan, V.K., Hariharan, A. and Yanny, H.F. 2007. Opioid-free anaesthesia: fracture neck of femur (DHS) surgery. Reg. Anesth. Pain Med. 32(Suppl. 1), 142.

Kaur, S., Saroa, R. and Aggarwal, S. 2015. Effect of intraoperative low-dose ketamine infusion of management of postoperative analgesia. J. Nat. Sci. Biol. Med. 6(2), 378-382.

Kissin, I., Brown, P.T., Robinson, C.A. and Bradley, E.L. 1991. Acute tolerance in morphine analgesia: continuous infusion and single injection in rats. Anesthesiology 74, 166-171.

Korpela, R., Korvenoja, P. and Meretoja, O. 1999. Morphine-sparing effect of acetaminophen in pediatric day-case surgery. Anesthesiology 8(91), 442-447.

Krystal, J.H., Karper, L.P., Seibyl, J.P., Freeman, G.K., Delaney, R., Bremner, J.D., Heninger, G.R., Bowers, M.B. and Charney, D.S. 1994. Subanesthetic Effects of the Noncompetitive NMDA Antagonist, Ketamine, in Humans. Arch. Gen. Psychiatry 51(3), 199-214.

Kuo, W.C. and Keegan, R.D. 2003. Comparative cardiovascular, analgesic, and sedative effects of medetomidine, medetomidine-hydromorphone, and medetomidine-butorphanol in dogs. Am. J. Vet. Res. 65(7), 931-937.

Lee, M., Silverman, S., Hansen, H., Patel, V. and Manchikanti, L. 2011. A Comprehensive review of Opioid-Induced Hyperalgesia. Pain Physician 14, 145-161.

Levite, M. 2012. The effects of opioids on immune cells, functions and diseases in Nerve Driven Immunity. Ed Ninkovic, J., Sabita, R., Springer Vienna, pp: 175-202.

Mansour, M.A., Mahmoud, A.A. and Geddawy, M. 2013. Non-opioid versus opioid-based general anaesthesia technique for bariatric surgery: A randomized double-blind study. Saudi J. Anaesth. 7(4), 387-391.

Martin, W.R. and Eades, C.G. 1961. Demonstration of tolerance and physical dependence in the dog following a short-term infusion of morphine. J. Pharmacol. Exp. Ther. 133, 262-270.

Mather, S.J. and Peutrell, J.M. 1995. Postoperative morphine requirement, nausea and vomiting following anaesthesia for tonsillectomy. Comparison of intravenous morphine and nonopioid analgesic techniques. Paediatr. Anaesth. 5, 185-188.

Maund, E., McDaid, C., Rice, S., Wright, K., Jenkins, B. and Woolacott, N. 2011. Paracetamol and selective and non-selective non-steroidal antiinflammatory drugs for the reduction in morphinerelated side-effects after major surgery: a systematic review. Br. J. Anaesth. 106(3), 292-297.

Mburu, D.N., Mbugua, S.W., Skoglung, L.A. and Lokken, P. 1988. Effects of paracetamol and acetylsalicylic acid on the post-operative course after experimental orthopaedic surgery in dogs. J. Vet. Pharmacol. Ther. 11(2), 163-171.

McCarthy, G.C., Megalla, S.A. and Habib, A.S. 2010. Impact of intravenous lidocaine infusion on postoperative analgesia and recovery from surgery: a systematic review of randomized controlled trials. Drugs 70(9), 1149-1163.

Mulier, J.P. 2016. Perioperative opioids aggravate obstructive breathing in sleep apnea syndrome: mechanisms and alternative anesthesia strategies. Curr. Opin. Anaesthesiol. 29(1), 129-133.

Odunayo, A., Podam, J.R., Kerl, M.R., DeClue and A.E. 2010. State-of-the-Art-Review: Immunomodulatory effects of opioids. J. Vet. Emerg. Crit. Care 20(4), 376-385.

Pasero, C. and McCaffery, M. 2012. Opioid-induced hyperalgesia. J. Perianesth. Nurs. 27(1), 46-50.

Peltoniemi, M.A., Hagelberg, N.M., Olkkola, K.T. and Teijo, S.I. 2016. Ketamine: A Review of Clinical Pharmacokinetics and Pharmacodynamics in Anesthesia and Pain Therapy. Clin. Pharmacokinet 55(9), 1059-1077.

Rang, H.P., Dale, M.M., Ritter, J.M., Flower, R.J. and Henderson, G. 2012. Rang and Dale's Pharmacology $7^{\text {th }}$ Edition. Elsevier, Spain.

Reid, J., Nolan, A.M., Hughes, J.M.L., Lascelles, D., Pawson, P. and Scott, E.M. 2007. Development of the short-form Glasgow Composite Measure pain scale (CMPS-SF) and derivation of analgesic intervention score. Anim. Welf. 16, 97-104.

Remy, C., Marret, E. and Bonnet, F. 2005. Effects of acetaminophen on morphine side-effects and consumption after major surgery: meta-analysis of randomized controlled trials. Br. J. Anaesth. 94(4), 505-513. 
Romano, M., Portela, D.A., Breghi, G. and Otero, P.E. 2015. Stress-related biomarkers in dogs administered regional anaesthesia or fentanyl for analgesia during stifle surgery. Vet. Anaesth. Analg. 43(1), 44-54.

Ronald, V.H. and Kissin, I. 1998. Rapid Development of Tolerance to Analgesia During Remifentanil Infusion in Humans. Anesth. Analg. 86(6), 13071311.

Sacerdote, P. 2006. Opioids and the immune system. Palliat. Med. 20, 9-15.

Sinatra, R.S., Jahr, J.S., Reynolds, L.W., Viscosi, E. R., Groudine, S.B. and Payen-Champenis, C. 2005. Efficacy and Safety of Single and Repeated Administration of 1 Gram Intravenous Acetaminophen Injection (Paracetamol) for Pain
Management after Major Orthopedic Surgery. Anesthesiology 102, 822-831.

Sinclair, M.D. 2003. A review of the physiological effects of $\alpha 2$-agonists related to the clinical use of medetomidine in small animal practice. Can. Vet. J. 44(11), 885-897.

Sukhani, R., Vazquez, J. and Pappos, A.L. 1996. Recovery after propofol with and without intraoperative fentanyl in patients undergoing ambulatory gynecologic laparoscopy. Anesth. Analg. 83, 975-981.

Ziemann-Gimmel, P., Goldfarb, A.A., Koppman, J. and Marema, R.T. 2014. Opioid-free total intravenous anaesthesia reduces postoperative nausea and vomiting in bariatric surgery beyond triple prophylaxis. Br. J. Anaesth. 112(5), 906-911. 